\title{
Inefficient A-to-I RNA editing and ALS
}

\author{
Shin Kwak, M.D.
}

\section{（臨床神経 2010;50:978）}

Key words : amyotrophic lateral sclerosis, ALS, glutamate recepter, RNA editing, excitotoxicity neuronal death, AMPA receptor

GluR2 is a subunit of the AMPA receptor, and failure of naturally occurring adenosine to inosine (A-to-I) conversion at its $\mathrm{Q} / \mathrm{R}$ site results in an increase of $\mathrm{Ca}^{2+}$ influx through the AMPA receptor, thereby inducing exaggerated neuronal excitation. Indeed, mutant mice unable to edit this site die from status epilepticus. A-to-I conversion at this site is inefficient in the dying motor neurons of sporadic ALS patients, whereas it is preserved in neurons of normal subjects and patients with other neurological diseases. Because adenosine deaminase acting on RNA 2 (ADAR2) specifically catalyzes the A-to-I conversion at this site, a reduction of ADAR2 expression may be involved in the pathogenesis of sporadic ALS.

To explore whether reduced ADAR2 activity is a direct cause of inefficient GluR2 Q/R site-editing and neuronal death, we generated genetically modified mice in which the $A D A R 2$ gene was conditionally targeted in motor neurons using the Cre-loxP system. ADAR2 was targeted in about $50 \%$ of motor neurons in the mutant mice, and the ADAR2lacking neurons were unable to edit the GluR2 Q/R site and hence expressed only $\mathrm{Q} / \mathrm{R}$ site-unedited GluR2. The conditional knockout mice were viable but displayed progressive motor dysfunction. Virtually all the ADAR2-lacking motor neurons disappeared by 12 months of age, remaining ADAR 2-expressing neurons healthy. Therefore, reduced ADAR2 activity with resultant inefficient RNA editing at the GluR2 Q/R site is a cause of slow death of motor neurons in sporadic ALS. The results will provide a clue to develop novel therapeutic strategy for sporadic ALS patients. 